\title{
Nanostructured Materials for Optoelectronic Applications
}

\author{
N.V. Kamanina ${ }^{a, *}$, P.Ya. Vasilyev ${ }^{a}$, S.V. Serov ${ }^{a}$, V.P. Savinov ${ }^{a}$, K.Yu. Bogdanov \\ AND D.P. USKOKOVIC ${ }^{c}$ \\ ${ }^{a}$ Vavilov State Optical Institute, 12 Birzhevaya Line, St. Petersburg, 199034, Russia \\ ${ }^{b}$ State Educational Institute Lyceum No. 1186, Moscow, Russia \\ ${ }^{c}$ Institute of Technical Sciences of the SASA, Belgrade, Serbia
}

\begin{abstract}
Study and optimization of new nanoscale materials useful for optoelectronic application have been considered. The search for the effective nanostructured materials has been revealed in two directions: to optimize the mechanical hardness of the inorganic systems and to increase the photorefractive parameters of the organics with nanoobjects. It has been testified that the surface mechanical properties of the inorganic materials via nanotubes treatment process can be drastically improved. For example, the surface mechanical hardness of the UV and IR range soft materials can be increased up to 3-10 times under the conditions of oriented nanotubes placement. It has been obtained that the nonlinear optical characteristics (nonlinear refraction $n_{2}$ and cubic nonlinearity $\chi^{(3)}$ ) of the organics thin films sensitized with fullerenes or nanotubes can be increased up to 3-4 orders of magnitude in comparison with the same parameters for bulk materials traditionally used for nonlinear optics.
\end{abstract}

PACS numbers: 42.70.-a, 42.79.-e, 78.20.-e, 78.30.Na

\section{Introduction}

It is well know that fullerenes and carbon nanotubes can be considered as the good candidates to improve the structural, mechanical, spectral, optics and nonlinear optics features of the different inorganic and organic materials operated in the UV, VIS and IR spectral ranges [1-3]. These nanoobjects have unique energy levels, high value of electron affinity energy, and strong hardness of their $\mathrm{C}-\mathrm{C}$ bonds. Moreover, the carbon nanotubes imaginary part of the dielectric constant is close to zero in the near and middle infrared spectral range that permits to save or improve the spectral properties of the matrix materials after nanoobjects treatment.

In the current paper some way to improve or optimize the mechanical and photorefractive properties of the inorganic and organic materials via fullerene or nanotubes treatment have been shown.

\section{Experimental conditions}

Some materials of the UV and IR spectral range, such as $\mathrm{MgF}_{2}$, as well as semiconducting structures and metals have been chosen as inorganic matrixes. The single wall carbon nanotubes have been used as promising nanoobjects. These nanotubes have been placed on the material surface using IR $\mathrm{CO}_{2}$-laser with $p$-polarized irradiation at wavelength of $10.6 \mu \mathrm{m}$ and power of $30 \mathrm{~W}$. Moreover, when nanotubes have been placed at the materials interface, the electric field close to $100-200 \mathrm{~V} \mathrm{~cm}^{-1}$

* corresponding author; e-mail: nvkamanina@mail.ru has been applied in order to orient the nanotubes during the deposition. It should be mentioned, that some structures have been additionally treated with surface electromagnetic waves (SEW) in order to obtain homogeneous surface with smallest roughness. The spectral characteristics of the nanotubes-treated materials have been tested using Perkin-Elmer Lambda 9 instrument. Surface mechanical hardness has been revealed using the CM-55 instrument, when the test has been made applying the silicon glass $\mathrm{K} 8$ as etalon. This etalon permits to obtain abrasive hardness close to zero at 3000 cycle with forces on indenter close to $100 \mathrm{~g}$.

The thin films of some conjugated polymers doped with fullerenes or nanotubes have been used as organics matrixes. The concentrations of fullerene sensitizers have been varied from 0.05 to $1.0 \mathrm{wt} . \%$ relative to the photosensitive organic component. The nanotubes concentration has been close to $0.05-0.1$ wt.\%. The film thickness has been varied within 1-4 micrometers. The photorefractive characteristics have been studied using four-wave mixing technique. The second harmonic of nanosecond pulsed Nd-laser at wavelength of $532 \mathrm{~nm}$ has been used. The energy density has been chosen in the range of $0.1-0.9 \mathrm{~J} \mathrm{~cm}^{-2}$. The amplitude-phase thin gratings have been recorded under Raman-Nath diffraction conditions at spatial frequency of 90 and $150 \mathrm{~mm}^{-1}$.

\section{Results and discussion}

\subsection{Direction 1.}

Search for the new nanostructures operated in the broad spectral range, including deep UV and middle IR, and having good mechanical and laser properties is the 
complex task. Many scientific and technological groups have made some steps to reveal the improved character- istics of such type of the materials. Our own steps in this direction have been firstly shown in paper [4].

TABLE I

Surface mechanical hardness of some materials after nanotubes deposition.

\begin{tabular}{|c|c|c|}
\hline Structures & $\begin{array}{l}\text { Abrasive surface hardness } \\
\text { (number of cycles before } \\
\text { visualization of the powder } \\
\text { from surface) }\end{array}$ & Remarks \\
\hline Pure organic glasses & 200-400 cycles & \multirow{4}{*}{$\begin{array}{l}\text { CM-55 instrument has been used. The test } \\
\text { has been made using silicon glass K8 as } \\
\text { etalon. This etalon permits to obtain abra- } \\
\text { sive hardness close to zero at } 3000 \text { cycle with } \\
\text { forces on indenter close to } 100 \mathrm{~g} \text {. }\end{array}$} \\
\hline organic glasses + nanotubes & $1500-3000$ cycles & \\
\hline $\mathrm{MgF}_{2}$ & 1000 cycles & \\
\hline $\mathrm{MgF}_{2}+$ nanotubes & 3000 cycles & \\
\hline $\mathrm{ZnSe}$ & 2500 cycles & \\
\hline ZnSe + nanotubes & 2700-3000 cycles & \\
\hline
\end{tabular}

TABLE II

AFM results to estimate the roughness of nanostructured $\mathrm{MgF}_{2}$.

\begin{tabular}{l|l|l|l|l}
\hline \hline Parameters & Materials & $\begin{array}{l}\text { Roughness before } \\
\text { nanotreatment }\end{array}$ & $\begin{array}{l}\text { Roughness after } \\
\text { nanotreatment }\end{array}$ & Remarks \\
\hline$R_{\mathrm{a}}$ & $\mathrm{MgF}_{2}$ & 6.2 & 2.7 & $\begin{array}{l}\text { The area of } 5000 \times 5000 \mathrm{~nm} \\
\text { has been studied via AFM } \\
\text { method }\end{array}$ \\
\cline { 2 - 5 } & $\mathrm{Si}$ & 0.2 & 0.170 & $\begin{array}{l}\text { The area of } 200 \times 200 \mathrm{~nm} \\
\text { has been studied via AFM } \\
\text { method }\end{array}$ \\
\hline$S_{\mathrm{q}}$ & $\mathrm{MgF}_{2}$ & 8.4 & 3.6 & $\begin{array}{l}\text { The area of } 5000 \times 5000 \mathrm{~nm} \\
\text { has been studied via AFM } \\
\text { method }\end{array}$ \\
\cline { 2 - 5 } & $\mathrm{Si}$ & 0.25 & 0.22 & $\begin{array}{l}\text { The area of } 200 \times 200 \mathrm{~nm} \\
\text { has been studied via AFM } \\
\text { method }\end{array}$ \\
\hline
\end{tabular}

It should be noticed that magnesium fluoride has been considered as a good model system. For this structure the spectral characteristics, atomic force microscopy data, measurements to estimate the hardness and roughness have been found in good connection. The main aspect has been made on interaction between nanotubes (their $\mathrm{C}-\mathrm{C}$ bonds) placed at the $\mathrm{MgF}_{2}$ surface via covalent bonding [5]. Table I presents the results of surface mechanical hardness of $\mathrm{MgF}_{2}$ structure after nanotubes placement; Table II shows the decrease of $\mathrm{MgF}_{2}$ roughness. $R_{\mathrm{a}}$ is the parameter of roughness, which corresponds to the relations between the number of ridges (baffle) above and under of the middle level of surface. $S_{\mathrm{q}}$ is the root-mean-square roughness. One can see from Table I that the nanostructured samples reveal the better surface hardness. For example, after nanotubes placement at the $\mathrm{MgF}_{2}$ surface, the surface hardness has been better up to 3 times in comparison with sample without nanoobjects. It should be noticed that for the organic glasses this parameter can be increased up to one order of magnitude. Moreover, the roughness of the $\mathrm{MgF}_{2}$ covered with nanotubes and treated with SEW has been improved essentially. Really, $R_{\mathrm{a}}$ and $S_{\mathrm{q}}$ roughness characteristics have been decreased up to three times. One can see from Table II that the deposition of the oriented nanotubes on the materials surface and SEW treatment decreases the roughness dramatically. Indeed this process is connected with the nature of the pure materials; it depends on the crystalline axis and the defects in the volume of the materials.

In order to explain observed increase of mechanical hardness we compared the forces and energy to bend and to remove the nanotubes, which can be connected with magnesium fluoride via covalent bond $\mathrm{MgC}$. Thus, the full energy responsible for destruction of the surface with nanotubes should be equal to the sum of $W_{\text {rem }}$ (energy to 
remove the layer of nanotubes) and of $W_{\text {destr }}$ (energy to destroy the magnesium fluoride surface). Due to the experimental fact that nanotubes covering increases drastically the surface hardness of $\mathrm{MgF}_{2}$ [5], the values of $W_{\text {rem }}$ and $W_{\text {destr }}$ can be close to each other. Under the conditions of the applied forces parallel to the surface, in order to remove the nanotubes from $\mathrm{MgF}_{2}$ surface, firstly, one should bend these nanotubes, and secondly, remove these nanotubes. In this case $W_{\text {rem }}$ are consisted of $W_{\text {elast }}$ (elasticity energy of nanotube) plus $W_{\mathrm{MgC}}$ (energy to destroy the covalent $\mathrm{MgC}$ binding). The energy of elasticity can be estimated as follow:

$$
W_{\text {elast }}=F_{\text {rem }}^{2} L^{3} / 6 E I
$$

where $E=1.5 \mathrm{TP}[3,6]$ is the modulus of elasticity, $I=\pi r^{3} \Delta r$ - is the inertion moment of the nanotube cross section at its wall thickness $\Delta r=0.34 \mathrm{~nm}$, $r=4 \mathrm{~nm}$; and $L=50 \mathrm{~nm}$ is the nanotube length. The force $F_{\text {rem }}$ can be estimated as follows:

$$
F_{\text {rem }}=F_{\mathrm{MgC}} 2 r / L,
$$

where $F_{\mathrm{MgC}}$ is close to $2 n N$.

Based on our calculation we might say that in order to broke the relief with nanotubes, we should firstly bend the nanotubes with energy that is 5 times more than the one, which can be applied to simply remove the nanotubes from surface after the destroying $\mathrm{MgC}$ binding. This fact is in good connection with the experimental results.

This calculation can be used to explain the results of dramatically increased mechanical surface hardness of the $\mathrm{MgF}_{2}$ covered with nanotubes. The experimental data testified that the surface mechanical hardness of $\mathrm{MgF}_{2}$ materials covered with nanotubes can be compared with the hardness of etalon based on silicon glass K8.

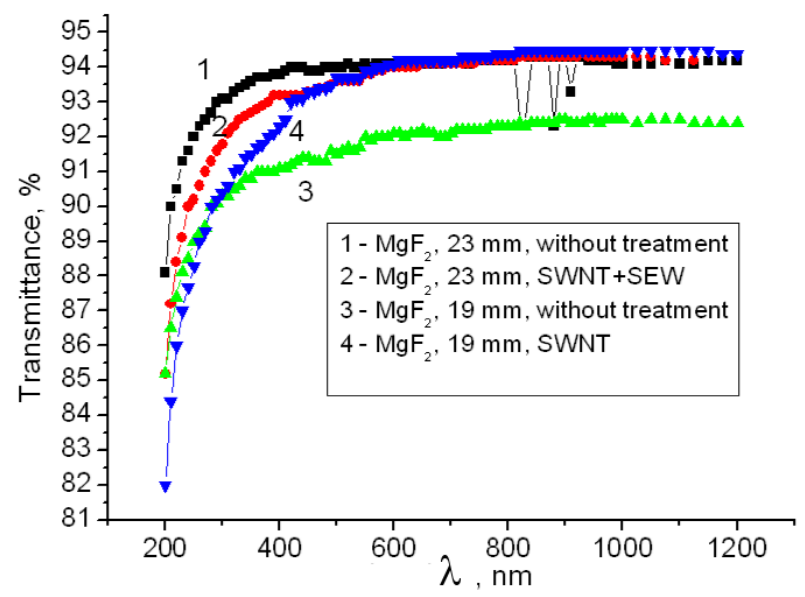

Fig. 1. UV-near IR spectra of $\mathrm{MgF} 2$ after single wall nanotubes (SWNT) deposition and after surface electromagnetic wave (SEW) treatment.

As a result of this process, the refractive index can be modified which explains the increase in transparency in the UV. Moreover, the spectral range saving or increasing in the IR range can be explained basing on the fact that the imaginary part of dielectric constant of carbon nanotubes, which is responsible for the absorption of the nanoobjects, is minimum (close to zero) in the IR range. The UV-VIS and near IR-spectra of the magnesium fluoride is shown in Fig. 1. It should be noticed that the drastic increase in the transparency at wavelength of $126 \mathrm{~nm}$ has been observed.

Really, for the 5 units of sample of $\mathrm{MgF}_{2}$, the transmittance $T$ has been increased after nanotubes deposition as arrow indicates: sample No $1 . T=61.8 \% \rightarrow T=66.6 \% 1$ No 2. $T=63.6 \% \rightarrow T=69 \%$; No 3 . $T=54.5 \% \rightarrow$ $T=65.8 \%$; No $4 . T=58.1 \% \rightarrow T=67.5 \%$; No 5 . $T=50.9 \% \rightarrow T=65 \%$.

\subsection{Direction 2.}

Table III presents the comparative data [7-8] of laser-induced changes in the refractive index of organics based on polyimides (PIs), $N$-(4-nitrophenyl)- $(L)$-prolinol, 2-( $N$-prolinol)-5-nitropyridine compounds for the previous experiments and the current ones. Laser-induced change in the refractive index $\Delta n_{i}$ has been calculated via Eq. (3.1). It has been based on measurement of the diffraction efficiency $\eta$ and calculation of $\Delta n_{i}$ via [9]:

$$
\eta=I_{1} / I_{0}=\left(\pi \Delta n_{i} d / 2 \lambda\right)^{2},
$$

where $I_{0}$ and $I_{1}$ - are intensities in the zero and in the first diffraction orders, $d$ - is the thickness of the media, and $\lambda$ - is the wavelength of the laser irradiation. Figure 2 demonstrates the distribution of the diffraction orders in the focal plate of the lens behind the organic thin film with nanoobjects.

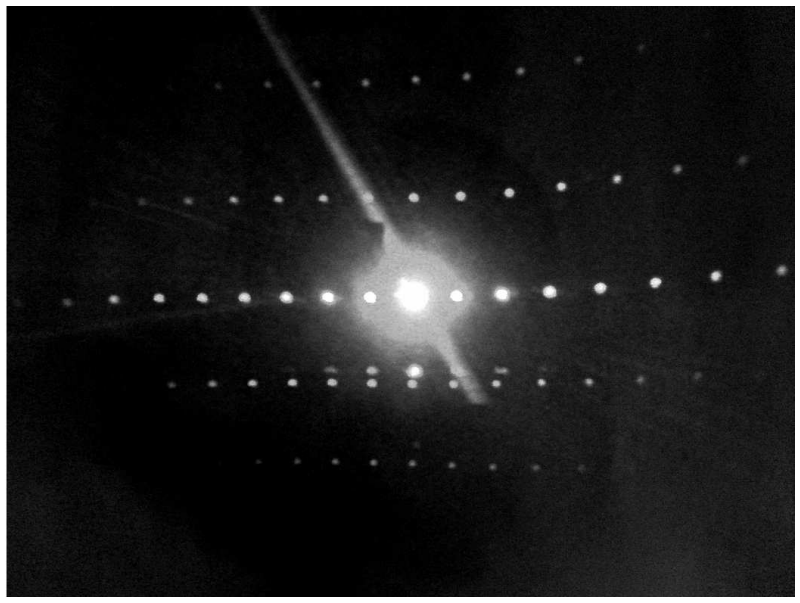

Fig. 2. Distribution of the diffraction orders when organics films with nanoobjects have been irradiated at spatial frequency of $100 \mathrm{~mm}^{-1}$.

One can see from Table III, that fullerene and nanotubes sensitization provokes essential change of the refractive index due to efficient charge transfer between organics molecules donor fragment and nanosensitizers. Moreover, it should be mentioned that at lower value of 


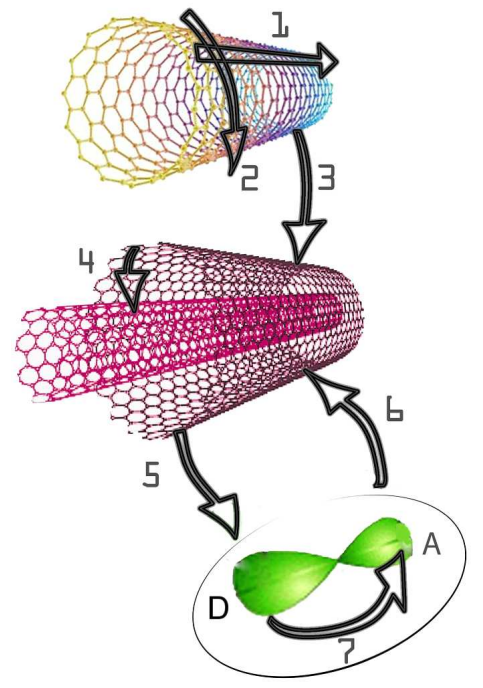

Fig. 3. Possible scheme of charge carrier moving in the organics media with nanotubes.

energy density $\left(0.3 \mathrm{~J} \mathrm{~cm}^{-2}\right.$ in comparison with previous ones of $0.6 \mathrm{~J} \mathrm{~cm}^{-2}$ ) and at larger spatial frequency $\left(\Lambda=150 \mathrm{~mm}^{-1}\right.$ in comparison with spatial frequency of $\Lambda=90 \mathrm{~mm}^{-1}$ ) the following values of laser-induced changes in the refractive index $\Delta n_{i}$ have been obtained: $5.0 \times 10^{-3}$ - for the polyimide sample with concentration of the nanotubes of $0.07 \mathrm{wt} . \%$; and $5.5 \times 10^{-3}$ - for the sample with concentration of the nanotubes of 0.1 wt.\%.
This fact can be explained by the additional mechanisms involved in photorefractive processes and connected with the charge carrier moving of odd nanotubes electrons in comparison with fullerenes ones. Really, we should take into account at least 7 ways for the charge carrier moving: 1 - along the nanotubes, 2 - across the nanotubes, 3 - between the nanotubes, 4 - inside the nanotubes if they are the multiwall ones, 5 and $6-$ between the organic conjugated molecules and nanotubes under the conditions of different relation of electron affinity energy of the organic molecules and nanotubes, 7 - inside the organic molecules, when the donor-acceptor interaction have been revealed. The possible scheme of this interaction is shown in Fig. 3. It should be noticed that the photoconductive parameters of the PI and other studied systems with fullerenes and nanotubes have been correlated with the nonlinear optical ones. It should be noticed that the increase in the dark photocurrent, for example PI, with concentration of the nanotubes close to $0.07 \mathrm{wt} . \%$, has been larger than that studied before for the pure PI or PI with fullerenes. The value of the dark current for the pure PI film is close to $10^{-13}$ A. Thus, more than $10^{4}-10^{5}$ times increase in the current has been found for the PI structures doped with nanotubes at the same level of bias voltage. A little bit larger value of the current has been revealed for the PI with $0.1 \mathrm{wt} . \%$ of the nanotubes. It should be mentioned, that current-voltage dependences have been connected with the laser induced change in the refractive index for the PI systems doped with nanotubes.

Comparative data of laser-induced change in the refractive index.

\begin{tabular}{|c|c|c|c|c|c|c|c|}
\hline $\begin{array}{l}\text { Structure studied at the spatial } \\
\text { frequency }\end{array}$ & $\begin{array}{c}\text { Nano-objects } \\
\text { content } \\
\text { [wt.\%] }\end{array}$ & $\begin{array}{l}\text { Wavelength, } \\
{[\mathrm{nm}]}\end{array}$ & $\begin{array}{l}\text { Energy density, } \\
\qquad\left[\mathrm{J} \mathrm{cm}^{-2}\right]\end{array}$ & $\begin{array}{c}\text { Spatial } \\
\text { frequency, } \\
{\left[\mathrm{mm}^{-1}\right]}\end{array}$ & $\begin{array}{l}\text { Laser pulse } \\
\text { width, [ns] }\end{array}$ & $\begin{array}{l}\text { Laser-induced } \\
\text { change } \\
\text { in the refractive } \\
\text { index, } \Delta n\end{array}$ & References \\
\hline Pure polyimide & 0 & 532 & 0.6 & 90 & 20 & $10^{-4}-10^{-5}$ & [7] \\
\hline Polyimide + malachite green & 0.2 & 532 & $0.5-0.6$ & $90-100$ & $10-20$ & $2.87 \times 10^{-4}$ & [8] \\
\hline Polyimide $+\mathrm{C}_{60}$ & 0.2 & 532 & $0.5-0.6$ & 90 & $10-20$ & $4.2 \times 10^{-3}$ & [7] \\
\hline Polyimide $+\mathrm{C}_{70}$ & 0.2 & 532 & 0.6 & 90 & $10-20$ & $4.68 \times 10^{-3}$ & [7] \\
\hline Polyimide + nanotubes & 0.1 & 532 & $0.5-0.8$ & 90 & $10-20$ & $5.7 \times 10^{-3}$ & [7] \\
\hline Polyimide + nanotubes & 0.05 & 532 & 0.3 & 150 & 10 & $4.5 \times 10^{-3}$ & Current \\
\hline Polyimide + nanotubes & 0.07 & 532 & 0.3 & 150 & 10 & $5.0 \times 10^{-3}$ & Current \\
\hline Polyimide + nanotubes & 0.1 & 532 & 0.3 & 150 & 10 & $5.5 \times 10^{-3}$ & Current \\
\hline$N$-(4-nitrophenyl)- $(L)$-prolinol $+\mathrm{C}_{60}$ & 1 & 532 & 0.3 & 100 & $10-20$ & $1.65 \times 10^{-3}$ & Current \\
\hline 2 - $(N$-prolinol $)-5$-nitropyridine $+\mathrm{C}_{60}$ & 1 & 532 & 0.3 & 100 & $10-20$ & $0.8 \times 10^{-3}$ & Current \\
\hline
\end{tabular}

Based on the value of $\Delta n$ and using procedure from paper [10], the nonlinear refraction $n_{2}$ and third order nonlinearity (cubic nonlinearity $\chi^{(3)}$ ) have been found. The data show that these nonlinear estimated parameters are close to $10^{-10_{-}}-10^{-9} \mathrm{~cm}^{2} \mathrm{~W}^{-1}$ and $10^{-10_{-}}-10^{-9} \mathrm{esu}$, respectively. It should be noticed, that in the current experiments using four-wave mixing technique, the nonlinear refraction coefficient and cubic nonlinearity have been estimated via Eqs. (3.4) and (3.5):

$$
\begin{aligned}
& n_{2}=\Delta n_{i} / I \\
& \chi^{(3)}=n_{2} n_{0} c / 16 \pi^{2},
\end{aligned}
$$


refractive index of the media, $c$ - is the speed of the light.

One can see that nonlinear optical parameters of the nanoobjects-doped conjugated structures are larger than those obtained for traditional nonlinear systems, including inorganic balk $\mathrm{LiNbO}_{3}$ crystal, that permits to apply these materials as effective holographic recording element in passive and active modes, as efficient 3D-media for spatial light modulator and telecommunications schemes, as switchers and as nonlinear absorbers in the visible and in the near-infrared spectral ranges.

\section{Conclusion}

In conclusion, the influence of the nanoobjects based on fullerenes and carbon nanotubes on mechanical, spectral, photoconductive, and nonlinear optical features have been shown The dramatic increase of surface mechanical hardness has been observed via nanotubes placement under condition of spectral range saving or improvement. Based on four-wave mixing technique the comparative studies of the nonlinear refraction and cubic nonlinearities have been found for the pure organics films and fullerene and nanotubes doped ones. As the result of this investigation, new area of applications of the treated materials can be found in the optoelectronics and laser optics, for example, for development of transparent UV and IR window, for gas storage and solar energy accumulation, as well as in nonlinear optical field and in search for 3D-media.

\section{Acknowledgments}

This study has been partially supported by the grant from Vavilov State Optical Institute (named "Ogranka", 2008-2009), RFBR grant No 08-02-00966 (2008-2010), RFBR grant No 10-03-00916 (2010-2012), and ISTC Project \# IPP A-1484 (2007-2009).

\section{References}

[1] W. Krätschmer, L.D. Lamb, K. Fostiropoulos, Nature, 347, 354 (1990).

[2] J. Robertson, Material Today 7, 46 (2004).

[3] Ch. Pul, F. Owns, Nanotechnology, Technosphere, Moscow 2007, p. 119.

[4] N.V. Kamanina, P.Ya. Vasilyev, V.I. Studeonov, Yu.E. Usanov, Optical Zhournal 75, 83 (2008).

[5] N.V. Kamanina, P.Ya. Vasilyev, V.I. Studeonov, $O p$ tical Zhournal 75, 57 (2008).

[6] S. Namilae, N. Chandra, C. Shet, Chem. Phys. Lett. 387, 247 (2004).

[7] N.V. Kamanina, D.P. Uskokovic, Materials and Manufacturing Processes 23, 552 (2008).

[8] N.V. Kamanina, A. Emandi, F. Kajzar, A.-J. Attias, Mol. Cryst. Liq. Cryst. 486, 1043 (2008).

[9] R.J. Collier, C.B. Burckhardt, L.H. Lin, Optical Holography, Mir, Moscow 1973; p. 686.

[10] S.A. Akhmanov, S.Yu. Nikitin, Physical Optics, Moscow University Press, Moscow 1997, p. 498. 